\title{
Paced Auditory Serial Addition Functional Test
}

National Cancer Institute

\section{Source}

National Cancer Institute. Paced Auditory Serial Addition Functional Test. NCI Thesaurus. Code C115792.

A standardized rating scale developed by DMA Gronwall et al in 1977 to assess auditory information processing speed and flexibility, as well as calculation ability. It is also used as a cognitive function test to evaluate sustained and divided attention. The test involves the presentation of single digits every 3 seconds and a study subject must add each new dig it to the one immediately prior to it. Scoring of this test is based on the total number correct out of 60 possible answers. 